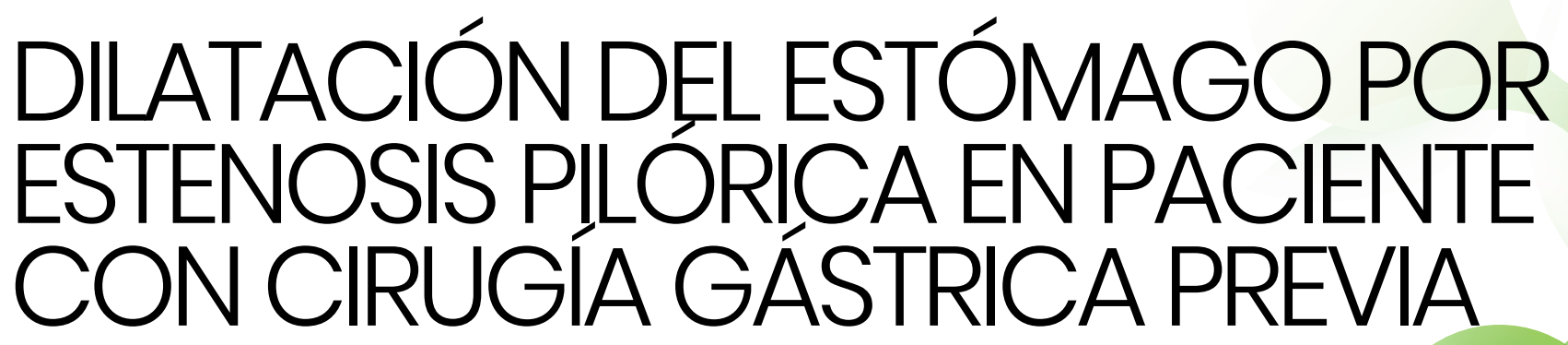

\title{
STOMACH DILATATION DUE PYLORIC STENOSIS PATIENT WITH PREVIOUS GASTRIC SURGERY
}

\section{Serrano Borrero I, Roldán Aviña JP, Merlo Molina S, Ferrusola Díaz AD, Muñoz Pozo F}

Hospital de Alta Resolución de Écija. Sevilla.

\section{Resumen}

Entre las complicaciones tardías de la cirugía gástrica podemos encontrar un amplio abanico de patologías, entre las que destaca el síndrome de obstrucción de la salida gástrica (SOSG). En los últimos 40-50 años las causas que lo producen han pasado de aquellas relacionadas con patología ulcerosa a las relacionadas con tumores malignos. Presentamos el caso de un paciente que acudió a urgencias por un cuadro de dolor abdominal asociado a un cuadro hiperemético, con antecedentes de cirugía gástrica unos 30 años antes, que presentaba un SOSG tratado mediante la colocación de un stent autoexpansible. Se revisa el diagnóstico diferencial y las diferentes opciones terapéuticas.

Palabras clave: dilatación estómago, estenosis pilórica, complicaciones cirugía gástrica.

\section{CORRESPONDENCIA}

Juan Pastor Roldán Aviña

Hospital de Alta Resolución de Écija

41.400 Écija, Sevilla

iproldan@aecirujanos.es

Fecha de envio: 23/03/2020

Fecha de aceptación: 23/04/2021
Abstract

Among late complications of gastric surgery we can find a wide range of pathologies, among which the gastric outlet obstruction (GOO) syndrome stands out. In the last 40-50 years, causes that produce it have changed from those related to ulcer pathology to those related to malignant tumors. We present the case of a patient who came to the emergency room with abdominal pain associated with a hyperemetic condition, with a previous gastric surgery 30 years previously, who presented GOO treated by placing a self-expanding stent. The differential diagnosis and the different therapeutic options are reviewed.

Keywords: stomach dilatation, pyloric stenosis, gastric surgery complications.

\section{Introducción}

La inflamación aguda o crónica del duodeno, puede conducir a un síndrome de obstrucción de la salida gástrica de tipo mecánico, conocido en inglés como "gastric outlet obstruction", y que se manifiesta en forma de dolor epigástrico postprandial, retraso del vaciamiento gástrico, anorexia, náuseas y vómitos. Cuando la situación se mantiene durante un periodo prolongado de tiempo, el estómago continúa dilatándose progresivamente, perdiendo rápidamente su tono muscular lo que acentúa aún más la sintomatología1. 


\section{Caso Clínico}

Paciente de 89 años de edad. Sus antecedentes más destacados son intervención por patología ulcerosa gástrica (probable piloroplastia - no aporta informes) unos 30 años antes; anticoagulado con Sintrom por trombosis venosa profunda M.I.D. 3 meses antes.

El paciente refiere dolor abdominal generalizado de varios meses de evolución, acentuado en la última semana, con vómitos alimentarios en los últimos días, motivo por el que acudió a Urgencias. Hábito intestinal estreñido con una deposición de características normales esa misma mañana. No hay fiebre.

La exploración está afectada por el dolor, con mal estado general. El abdomen era blando, aunque poco depresible, timpanizado sobre todo en el hemiabdomen derecho. Existía una sensación de ocupación del cuadrante superior izquierdo del abdomen sin bazuqueo gástrico. Cicatriz de laparotomía media sin complicaciones.

Se prescribió tratamiento con Metamizol 2 g IV, Omeprazol $40 \mathrm{mg} \mathrm{IV}$, Metoclopramida $10 \mathrm{mg}$ IV. Tras un nuevo episodio de vómito, se colocó sonda nasogástrica.

En la analítica destacaba una anemia microcítica e hipocroma ya conocida con $\mathrm{Hb} 6,5 \mathrm{mg} / \mathrm{dl}$ y hematocrito $30,3 \%$ con recuento y fórmula leucocitaria normales. INR 2,47.

En la radiografía abdominal (Figura 1) llamaba la atención un desplazamiento hacia abajo y hacia los lados del contenido intestinal por una sombra de densidad agua que impresionaba como un estómago retencionista. Se completó el estudio con un TAC de abdomen (Figuras 2 y 3 ) que informó de una gran dilatación gástrica, con contenido en su interior (sonda en posición correcta) y masa de bordes mamelonados a nivel pilórico, estenosante, con un tamaño $43,7 \times 52 \mathrm{~mm}$ que provoca obstrucción gástrica, compatible con neoplasia pilórica, junto con adenopatías locorregionales sugestivas de afectación metastásica.
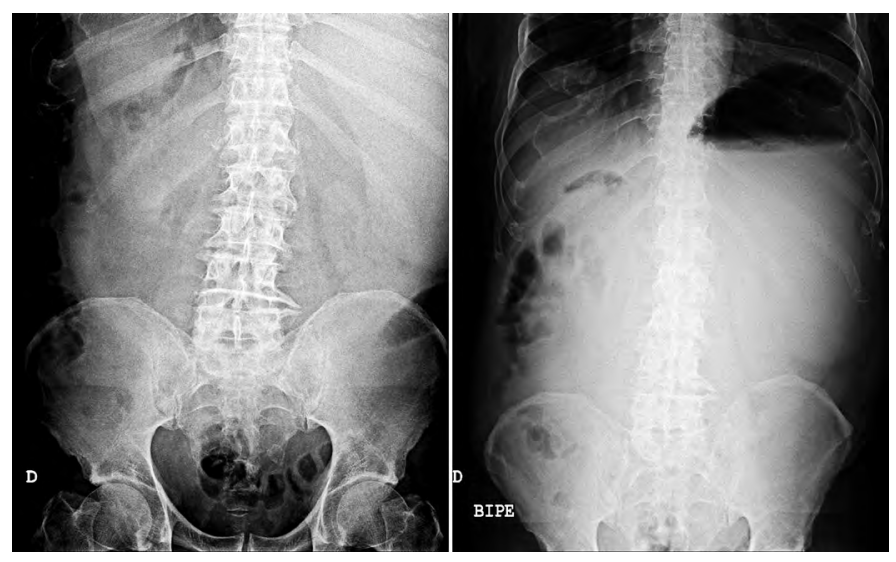

Figura 1

Radiografías de abdomen (proyecciones en decúbito supino y en bipedestación).

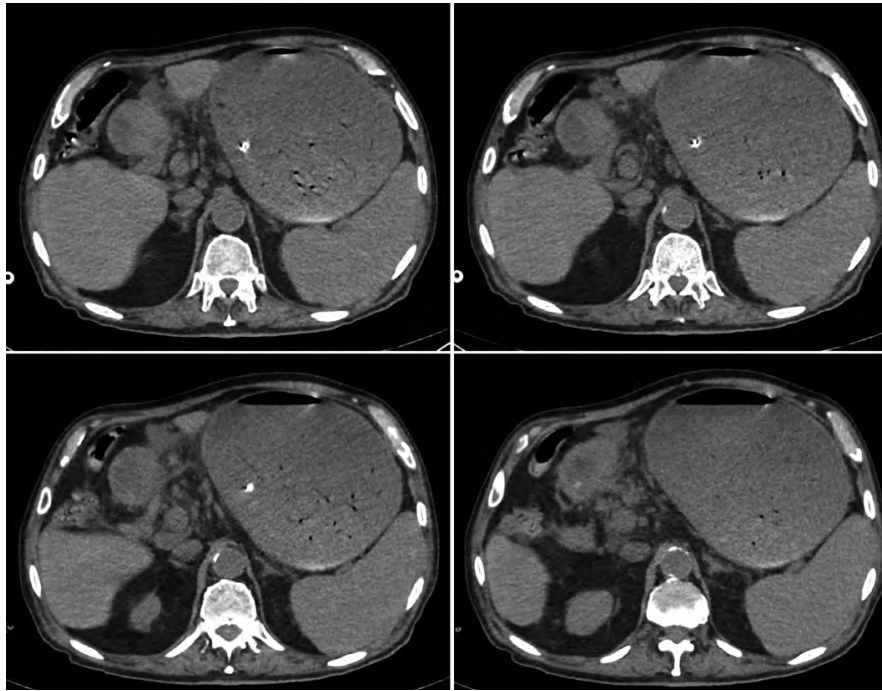

Figura 2

TAC de abdomen (cortes axiales).

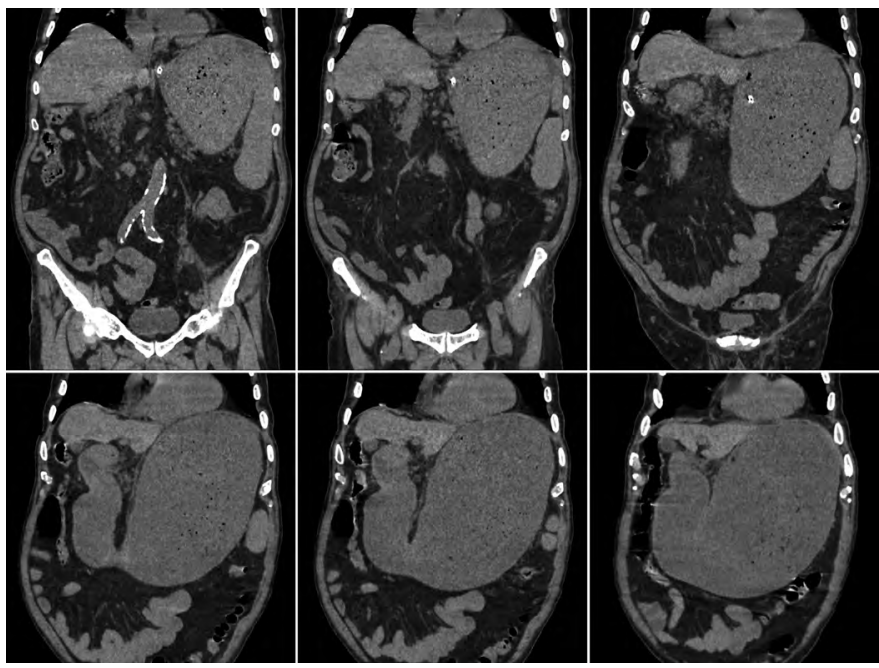

Figura 3

TAC de abdomen (cortes coronales).

Una vez ingresado, comenzó tratamiento con Nutrición Parenteral y se completaron los estudios con una endoscopia oral en la que el estómago sólo fue valorado en la zona de antro por la existencia de abundante contenido líquido/semisólido, que se intentó aspirar consiguiéndose solo en parte. La zona del píloro estaba desestructurada, con desaparición del orificio pilórico por la existencia de una mucosa protruyente que ocupaba toda la zona impidiendo el paso del endoscopio. El aspecto de la mucosa no era claramente neoplásico, pudiendo presentar ese aspecto por compresión extrínseca neoplásica a nivel de bulbo. Las biopsias tomadas informaban de una mucosa con cambios mínimos, sin identificarse tejido neoplásico.

El curso clínico fue favorable, con desaparición de los síntomas que motivaron su ingreso. Finalmente se completó el tratamiento con una endoscopia oral terapéutica en la que se apreciaba una estenosis de píloro, severa, que impedía el paso del endoscopio. Bajo control 
radiológico se consiguió ver, contrastando la luz, la existencia de una estenosis de unos 10-15 mm de longitud a nivel de bulbo. Bajo control endoscópico y radiológico se procedió a la colocación de una prótesis duodenal no recubierta de $22 \times 120 \mathrm{~mm}$ (Figura 4).

El paciente fue dado de alta tolerando dieta oral triturada, con tránsito intestinal normal y tras haber sido valorado por la Unidad de Cuidados Paliativos. Cuatro meses después de alta, el paciente continúa asintomático, con buena calidad de vida.

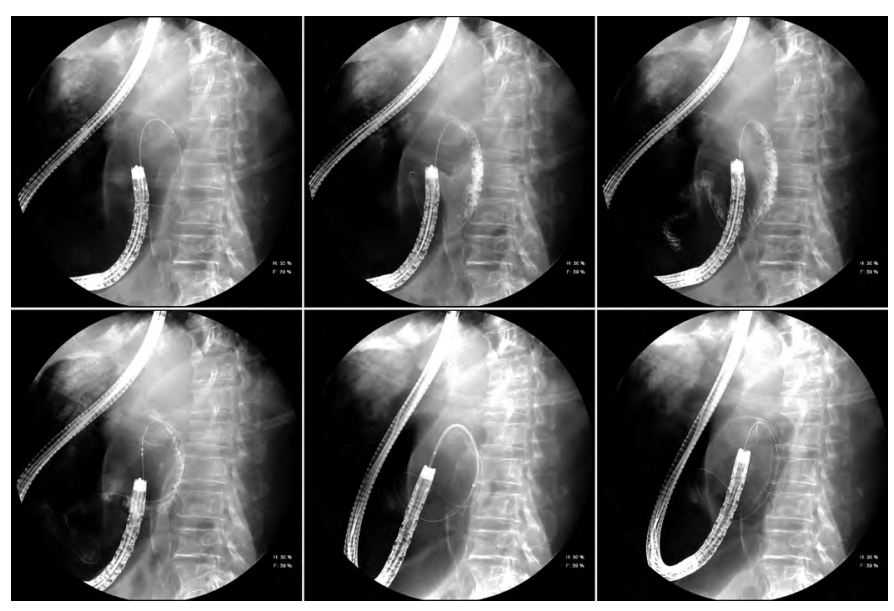

Figura 4

Control radiológico de la colocación de un stent autoexpansible.

\section{Discusión}

La obstrucción intrínseca o extrínseca del píloro o del duodeno es el mecanismo fisiopatológico del SOSG; la causa final de la obstrucción dependerá de la etiología subyacente. Debido a la gran diversidad de causas que lo pueden producir se desconoce la incidencia real de esta patología².

Los pacientes inicialmente presentan síntomas intermitentes que progresan hasta la obstrucción completa de la salida gástrica. Característicamente el vómito es el síntoma cardinal, presentado restos alimentarios ingeridos varios días antes. En la fase aguda o crónica de la obstrucción, los vómitos continuos pueden provocar deshidratación y anomalías electrolíticas. En cuadros de instauración lenta y progresiva es posible incluso que con una obstrucción severa el paciente presente mínimos síntomas sin afectación nutricional ${ }^{3}$.

Inicialmente, los pacientes pueden demostrar una mejor tolerancia a los líquidos que a los alimentos sólidos y en una etapa posterior, los pacientes pueden desarrollar una pérdida de peso significativa debido a una ingesta calórica deficiente. La desnutrición es un signo tardío, pero puede ser muy profunda en pacientes con malignidad concomitante.

Cuando la obstrucción persiste, los pacientes terminan presentando una dilatación gástrica significativa, perdiendo el estómago finalmente su contractilidad. Los alimentos no digeridos se acumulan aumentando el riesgo de presentar una neumonía por aspiración.
A finales del siglo $\mathrm{XX}$, las causas benignas constituían el $37 \%$ de los casos, y dentro de ellas la enfermedad ulcerosa duodenal complicada suponía el 90. Con el uso de fármacos inhibidores de la bomba de protones (IBP) y los tratamientos de erradicación de la infección por H. Pylori se ha reducido la incidencia de úlcera péptica, y las enfermedades malignas se han convertido en la principal causa del SOSG.

Aunque su incidencia es baja en comparación con otras patologías gástricas, el diagnóstico de dilatación gástrica debe ser tenido siempre en cuenta en pacientes con antecedentes de cirugía del tracto digestivo superior. Un diagnóstico precoz facilitará su correcto tratamiento médico y quirúrgico (si fuese necesario), disminuyendo la morbimortalidad asociada a este cuadro.

La endoscopia es el examen más útil para establecer la localización y la posible causa de la obstrucción de la salida gástrica y sirve para obtener muestras de tejido de las áreas implicadas para confirmar o excluir etiología maligna. Los estudios de contraste con bario o agentes hidrosolubles, así como la radiografía simple de abdomen pueden mostrar un estómago retencionista y aportar pistas sobre la enfermedad subyacente. La ausencia de paso de contraste al intestino delgado sugiere una obstrucción completa. EI TAC es útil especialmente para evaluar el grosor de la pared del píloro o del estómago, evaluar la existencia de adenopatías regionales, así como el área pancreática, biliar y el retroperitoneo ${ }^{5,6}$.

En el diagnóstico diferencial ${ }^{5}$, una vez realizada la endoscopia oral, contempla dos amplias categorías etiológicas: benignas o malignas, quedando recogidas en la Tabla 1.

\begin{tabular}{|c|c|c|}
\hline \multicolumn{3}{|c|}{$\begin{array}{l}\text { Tabla 1. Causas del Síndrome de } \\
\text { Obstrucción de la Salida Gástrica (SOSG). }\end{array}$} \\
\hline \multicolumn{2}{|c|}{ Enfermedad benigna } & $\begin{array}{c}\text { Enfermedad } \\
\text { maligna }\end{array}$ \\
\hline Úlcera péptica & Pancreatitis & Cáncer gástrico \\
\hline $\begin{array}{l}\text { Ingestión de } \\
\text { caústicos }\end{array}$ & Pólipos inflamatorios & Linfoma Gástrico \\
\hline $\begin{array}{l}\text { Estenosis inducida } \\
\text { por AINES }\end{array}$ & Causas infecciosas & Cáncer de páncreas \\
\hline $\begin{array}{l}\text { Síndrome de } \\
\text { Bouveret }\end{array}$ & $\begin{array}{l}\text { Gastroenteritis } \\
\text { tuberculosa }\end{array}$ & Cáncer de duodeno \\
\hline $\begin{array}{l}\text { Estenosis pilórica } \\
\text { hipertrófica }\end{array}$ & $\begin{array}{l}\text { Gastroenteritis por } \\
\text { CMV }\end{array}$ & Colangiocarcinoma \\
\hline latrogénico & Causas infiltrativas & $\begin{array}{l}\text { Cáncer de vesícula } \\
\text { biliar }\end{array}$ \\
\hline $\begin{array}{c}\text { Estenosis de cicatriz } \\
\text { o anastomosis } \\
\text { postquirúrgica }\end{array}$ & $\begin{array}{l}\text { Gastroenteritis } \\
\text { eosinofílica }\end{array}$ & Cáncer metastásico \\
\hline $\begin{array}{l}\text { Disección mucosa } \\
\text { endoscópica }\end{array}$ & $\begin{array}{l}\text { Enfermedad de } \\
\text { Crohn }\end{array}$ & \\
\hline
\end{tabular}

En cuanto al manejo terapéutico inicial, todos los pacientes deben ser hospitalizados para la inmediata reposición hidroelectrolítica y descompresión nasogástrica. En pacientes con 
obstrucción por enfermedad ulcerosa se aprecia una mejoría de los síntomas a las 48-72 horas, según las series revisadas, debido a la disminución del edema y espasmo gracias a los IBP y el uso de sondaje nasogástrico ${ }^{7}$. En cambio, en pacientes con síntomas de causa maligna debemos recurrir a métodos endoscópicos, (dilatación con balón o inserción de stents) cuando la única opción es paliativa y a la cirugía cuando se requieren resultados eficaces a largo plazo ${ }^{2}$. En cuanto a técnica quirúrgica se refiere, la resección quirúrgica y derivación (gastroyeyunostomia) es la opción más empleada.

El dilema se presenta ante pacientes con enfermedad avanzada donde se busca una actitud paliativa, debatiéndose entre el uso de stents autoexpansibles y la realización de gastroyeyunostomías. Parece ser que los stents aportan periodos de hospitalización más cortos, menor tiempo para la reintroducción de la ingesta oral y no hay diferencias significativas en cuanto a mortalidad, complicaciones y tasas de supervivencia cuando comparamos con cirugía, En contraposición, a veces se requieren procedimientos endoscópicos repetidos por los fallos de los stents ${ }^{8,9}$.

Como conclusión el SOSG no es una entidad única; es la consecuencia clínica y fisiopatológica de cualquier proceso patológico que produzca un impedimento mecánico al vaciamiento gástrico. Un retraso en la actuación puede comprometer el tejido gástrico ya edematizado y complicar la intervención quirúrgica previa, aumentando la tasa de morbimortalidad, disminuyendo la eficacia de los tratamientos.

\section{Bibliografía}

1. Tringali A, Giannetti A, Adler DG. Endoscopic management of gastric outlet obstruction disease. Ann Gastroenterol 2019; 32: 330-337.

2. Appasani S, Kochhar S, Nagi B, Gupta V, Kochhar R. Benign gastric outlet obstruction--spectrum and management. Trop Gastroenterol 2011; 32: 259266.

3. Al-Ghazal T, AL-Zahir AA, Al-Momen SA. Severe postoperative gastric outlet obstruction without nutritional deficiency. BMJ Case Rep 2019; 12: e231734.

4. Irani S, Khashab M. Gastric outlet obstruction: when you cannot do an endoscopic gastroenterostomy or enteral stent, try an endoscopic duodenojejunostomy or jejunojejunostomy. VideoGIE 2020; 5:125-128.

5. Wolf LL, Nijagal A, Flores A, Buchmiller TL. Late-onset hypertrophic pyloric stenosis with gastric outlet obstruction: case report and review of the literature. Pediatr Surg Int 2016; 32: 1013-1016.

6. Sukumar V, Ravindran C, Prasad RV. Demographic and Etiological Patterns of Gastric Outlet Obstruction in Kerala, South India. N Am J Med Sci 2015; 7:403406.

7. Koop AH, Palmer WC, Stancampiano FF. Gastric outlet obstruction: a red flag, potentially manageable. Clevel Clin J Med 2019; 86: 345-353.

8. Jeurnink SM, Polinder S, Steyerberg EW, Kuipers EJ, Siersema PD. Cost comparison of gastrojejunostomy versus duodenal stent placement for malignant gastric outlet obstruction. J Gastroenterol 2010; 45: 537-543.

9. Coda S, Oda I, Gotoda T, Yokoi C, Kikuchi T, Ono H. Risk factors for cardiac and pyloric stenosis after endoscopic submucosal dissection 